\title{
Quality Monitoring of Various Local and Imported Brands of Bottled Drinking Water in Saudi Arabia
}

\author{
Hisham A. Maddah, Abdulazez S. Alzhrani \\ Department of Chemical Engineering, King Abdulaziz University, Rabigh, Saudi Arabia \\ Email: hmaddah@kau.edu.sa
}

How to cite this paper: Maddah, H.A. and Alzhrani, A.S. (2017) Quality Monitoring of Various Local and Imported Brands of Bottled Drinking Water in Saudi Arabia. World Journal of Engineering and Technology, 5, 551-563.

https://doi.org/10.4236/wjet.2017.54047

Received: August 11, 2017

Accepted: September 10, 2017

Published: September 13, 2017

Copyright ( 92017 by authors and Scientific Research Publishing Inc. This work is licensed under the Creative Commons Attribution International License (CC BY 4.0). http://creativecommons.org/licenses/by/4.0/

\begin{abstract}
Minerals and constitutes in drinking water are vital for the nutrition of human bodies. Certain limits of water quality parameters must be met to ensure the safety of bottled drinking water for the human consumption. Thirty two local and twelve imported brands of bottled water in Saudi Arabia have been collected to verify their compliance with international and local standards which are EPA (2016), WHO (2013) and SASO (2009). A proposed scoring system is used to evaluate the water quality. Fayha and Hilwa brands have been selected as the best local brands whereas Volvic brand as the optimal imported brand in the western region of Saudi Arabia. The local water brands are more reliable to the standards than the imported brands. Licensed water brands in Saudi Arabia are found to have a good water quality which satisfied the quality requirements.
\end{abstract}

\section{Keywords}

Quality, Monitoring, Assessment, Standards, Drinking Water, Reliability

\section{Introduction}

Water has always been prioritized as a top necessity for human beings since it makes up to $70 \%$ of human total body weight. Thus, providing communities with clean potable water will increase their life qualities as well as the expected life-span [1]. Drinking or potable water is defined as purified water that is clean enough for human consumption with low immediate or long-term risks [2]. Water minerals play a significant role in predicting water quality; the importance of minerals to the nutrition of human bodies varies based on the mineral class [3]. There are two classes of minerals in water: Macro elements and trace 
elements. Macro elements are those minerals required in our diet in excess of 50 $\mathrm{mg} /$ day whereas trace elements are the minerals that are required in our nutrition less than $50 \mathrm{mg} /$ day. Study reports approved that there is a strong correlation between chronic diseases in humans and the presence of trace elements in potable water [4].

During the last two decades, various studies have been conducted for the assessment of the quality of potable water in Saudi Arabia [5] [6] [7]. In an earlier study, the concentrations of metals in nine bottled water brands in Saudi Arabia have been investigated and the results showed that there were higher concentrations of calcium and sodium in two brands than the values shown on their labels [5]. Another study claimed that the levels of total dissolved solids (TDS) and other minerals such as calcium, magnesium, sodium, potassium, nitrates, chloride and sulfate that were observed in fourteen domestic and seven imported brands in Saudi Arabia were within the safe limits of local and international standards [6]. However, a bromate study on fifty marketed bottled water brands in Saudi Arabia indicated that $70 \%$ of the water samples were not compatible with the United States Environmental Protection Agency (EPA) standard of 10 $\mu \mathrm{g} / \mathrm{L}$ and $60 \%$ of the samples were not accepted by the Saudi Arabia bromate standard of $25 \mu \mathrm{g} / \mathrm{L}$ [2].

Saudi Arabia registered a significant increase in water demand from bottled water due to the growing population and the scarcity of fresh water sources. Therefore, several new brands have been introduced in the market in order to supply enough bottled water to the community. However, to the best of our knowledge, there are no previous assessment studies on the water quality of these new brands in Saudi Arabia which could not be in compliance with the EPA, World Health Organization (WHO) and Saudi Arabian Standards Organization (SASO) standards [7].

The objective of this study is to investigate the physico-chemical characteristics of some of the local and imported brands of bottled drinking waters distributed and sold in Saudi Arabia. The accuracy of the chemical contents and their concentrations and other parameters printed on the manufacturer labels of different brands have been compared to the United States EPA, WHO and SASO standards. Thirty two local brands and twelve imported brands of bottled water in Saudi Arabia have been collected from domestic supermarkets within the western region for further investigations. The concentration of total dissolved solids (TDS), $\mathrm{pH}$, cations and anions average compositions and further analyses are carried out in the present work.

\section{Methodology}

Thirty two local and twelve imported bottled water brands were collected from different supermarkets within the western region from Jeddah, Makkah, Thuwal and Rabigh in Saudi Arabia. A total of forty four brands will be studied for their water qualities; the collected bottled water brand names are shown in Table 1. 
Table 1. Collected local \& imported bottled water brands from Saudi Arabia market.

\begin{tabular}{|c|c|c|}
\hline \multicolumn{2}{|c|}{ Local Brands } & \multirow{2}{*}{$\frac{\text { Imported Brands }}{\text { Evian }}$} \\
\hline $\mathrm{Al}$ Ain & Hada & \\
\hline Nova & Dala & Elite \\
\hline Fayha & Hayat & Roseland \\
\hline Panda & Hala & Tannourine \\
\hline Qtaf & Alabeer & Masafi \\
\hline Alwadi & Hilwa & Volvic \\
\hline Arwa & Aqua land & highland \\
\hline Nestle & Qarzal & Rim \\
\hline Safa & Najd & Hamidiye \\
\hline Berain & Najran & Oxygenizer \\
\hline Al-Qassim & Hajar & Fiji \\
\hline Tania & Rafa & Goccia blu \\
\hline Hana & Aloyoun & - \\
\hline Mozn & Lora & - \\
\hline Aquafina & Al Tharawat & - \\
\hline Farm Superstores & Bambini & - \\
\hline
\end{tabular}

Three official water quality standards have been used to study the compliance of the collected bottled water brands with respect to different quality parameters. The EPA and WHO international standards plus the SASO local standard of different water quality parameters are shown in Table 2.

Every bottled water brand is evaluated independently to determine the deviation of each parameter from the proposed three quality standards. A proposed scoring system from 0 to 5 point scale is used to select the best water brands, either local or imported, in the western region of Saudi Arabia. Zero (0) score means that there is no associated potential health risks or chronic diseases in humans with a certain parameter whereas five (5) score indicates a very critical parameter that is associated with adverse health risks such as cancer from excess amounts of chlorine $(\mathrm{Cl})$ and/or bromate $\left(\mathrm{BrO}_{3}\right)$ or common health problems like diarrhea, cathartic effects and dehydration from excess amounts of sulfate $\left(\mathrm{SO}_{4}\right)$ [10] [11] [12] [13].

Different local bottled water brands have been compared to recommend the best local brand in Saudi Arabia which met most of the three standards' parameters and got the highest score points. The same comparison is established between various imported water brands to identify the best imported water brand in Saudi Arabia that got the highest score. Additionally, the identified best local and imported brands have been also compared to determine the optimum and overall bottled water brand in Saudi Arabia that is in compliance with the studied international and local standards. Values of the water quality parameters 
Table 2. International and local bottled drinking water standards [2] [7] [8] [9].

\begin{tabular}{ccccc}
\hline Parameter & EPA $(2016)^{*}$ & WHO $(2013)^{*}$ & SASO $(2009)^{*}$ & Score $^{*}{ }^{* *}$ \\
\hline $\mathrm{pH}$ & $7.0-7.3$ & $6.5-8.5$ & $6.5-8.5$ & 3 \\
$\mathrm{TDS}(\mathrm{mg} / \mathrm{L})$ & 500 & 600 & 500 & 3 \\
$\mathrm{Ca}(\mathrm{mg} / \mathrm{L})$ & 140 & 100 & 200 & 1 \\
$\mathrm{Mg}(\mathrm{mg} / \mathrm{L})$ & - & 50 & 150 & 1 \\
$\mathrm{Na}(\mathrm{mg} / \mathrm{L})$ & 95 & 200 & 100 & 2 \\
$\mathrm{~K}(\mathrm{mg} / \mathrm{L})$ & 3.5 & 12 & - & 1 \\
$\mathrm{HCO}(\mathrm{mg} / \mathrm{L})$ & - & 125 & - & 0 \\
$\mathrm{SO} \mathrm{O}_{4}(\mathrm{mg} / \mathrm{L})$ & 250 & 250 & 150 & 4 \\
$\mathrm{Cl}(\mathrm{mg} / \mathrm{L})$ & 250 & 250 & 150 & 4 \\
$\mathrm{~F}(\mathrm{mg} / \mathrm{L})$ & 2 & 1.5 & 0.8 & 2 \\
$\mathrm{BrO}_{3}(\mathrm{mg} / \mathrm{L})^{* * *}$ & 0.01 & 10 & 0.025 & 3,4 or 5 \\
$\mathrm{Fe}(\mathrm{mg} / \mathrm{L})$ & 0.3 & 0.3 & 0.3 & 2 \\
\hline
\end{tabular}

${ }^{*}$ EPA: Environmental Protection Agency; WHO: World Health Organization; SASO: Saudi Arabian Standards Organization. ${ }^{* *} \mathrm{~A}$ scoring system is proposed as the following: 0 to 5 point scale where zero (0) means the parameter is not critical or important in water quality and has no potential health risks on humans and five (5) means that the parameter is very critical and certainly has adverse human health effects. ${ }^{*} *$ Since labels info are given in ppb and with no exact values, varied score points have been considered for Bromate to ensure the credibility of its critical effects in water quality; 3,4 , and 5 pts are given to $<10,<5$ and $0 \mathrm{ppb}$ of $\mathrm{BrO}_{3}$, respectively.

of different collected bottled water labels have been reported in Table 3 and Table 4 for local and imported brands, respectively.

Scoring system assumptions:

1) Score points of every parameter is in the scale of 0 to 5 and is determined by the best of the authors' knowledge and references.

2) Zero (0) score suggests no associated potential health risks or chronic diseases in humans with a certain parameter.

3) Five (5) score indicates a very critical parameter that is associated with adverse health risks.

4) Any brand parameter value that is below or equal to the standard value meets the standard and that brand gets the assigned score of that parameter as shown in Table 2.

5) Unknown parameter values are given zero (0) score points.

6) $\mathrm{NO}_{3}$ anion scores are not computed due to lack in $\mathrm{NO}_{3}$ international and local standards.

7) All $\mathrm{HCO}_{3}$ anion scores are zeroes since they do not pose noticeable health risks to humans.

8) The total score points of each brand is calculated from the summation of all the parameters' achieved points in the three water standards as shown in Equation (1). The normalized brand score is obtained from Equation (2).

9) $\mathrm{pH}$ values within a standard range or similar to the boundary standard values get three (3) score points. 
Table 3. Quality parameter values of local bottled drinking water brands.

\begin{tabular}{|c|c|c|c|c|c|c|c|c|c|c|c|c|c|c|}
\hline \multirow{2}{*}{ Brand\# } & \multirow{2}{*}{ Brand Name } & \multirow{2}{*}{$\mathrm{pH}$} & \multirow{2}{*}{$\begin{array}{c}\text { TDS } \\
(\mathrm{mg} / \mathrm{L})\end{array}$} & \multicolumn{5}{|c|}{ Cations Average Composition (ppm) } & \multicolumn{5}{|c|}{ Anions Average Composition (ppm) } & \multirow{2}{*}{$\begin{array}{l}(\mathrm{ppb}) \\
\mathrm{BrO}_{3}\end{array}$} \\
\hline & & & & $\mathrm{Na}$ & $\mathrm{K}$ & $\mathrm{Ca}$ & $\mathrm{Mg}$ & $\mathrm{Fe}$ & $\mathrm{Cl}$ & $\mathrm{NO}_{3}$ & $\mathrm{SO}_{4}$ & $\mathrm{HCO}_{3}$ & $\mathrm{~F}$ & \\
\hline 1 & Al Ain & $6.5-7.5$ & 105 & $<6$ & $<0.5$ & $<3$ & $<15$ & $<0.01$ & $<7$ & 1 & $<60$ & $<10$ & $<1.5$ & $<10$ \\
\hline 2 & Nova & 7.4 & 120 & 17 & 1.2 & 11 & 3.4 & - & 19 & 3 & 26 & 26 & 1 & - \\
\hline 3 & Fayha & 7.3 & 120 & 15 & 0.9 & 15 & 4 & 0.01 & 25 & 4 & 20 & 15 & 0.9 & $<5$ \\
\hline 4 & Panda & 7.2 & 130 & 29 & 1 & 6 & 2 & - & 32 & 4 & 30 & 25 & 1 & $<10$ \\
\hline 5 & Qtaf & 7 & 110 & 13 & 0.68 & 12 & 3 & 0.02 & 31 & 5 & 32 & 22 & 0.9 & $<10$ \\
\hline 6 & Alwadi & 7.5 & 116 & 24.6 & 0.8 & 2.4 & 0.5 & 0.02 & 30 & 5 & 12 & 40 & 0.75 & - \\
\hline 7 & Arwa & 6.8 & 121 & 3 & $<1$ & $<1$ & 21.1 & $<0.1$ & $<1$ & $<1$ & 74.5 & 7.7 & $0.8-1.2$ & $<10$ \\
\hline 8 & Nestle & 7.1 & 235 & 18 & 0.2 & 36 & 4.7 & $<0.02$ & 68 & $<1$ & 22 & 42 & 0.9 & $<10$ \\
\hline 9 & Safa & 7.2 & 110 & 13 & 0.2 & 12 & 1.5 & 0 & 26 & 0.04 & 7 & 28 & 1 & $<10$ \\
\hline 10 & Berain & 8 & 145 & 16 & 0.5 & 20 & 1 & 0.01 & 32 & 1.5 & 5 & 35 & 1 & $<5$ \\
\hline 11 & Al-Qassim & $6.85-7.4$ & $140-180$ & 25 & 1 & 12 & 2 & - & 35 & 5 & 25 & 10 & 0.95 & - \\
\hline 12 & Tania & 7.2 & 120 & 12.3 & 1.5 & 14.4 & 3 & 0 & 17.5 & 2 & 28 & 24 & 0.9 & $<10$ \\
\hline 13 & Hana & 7.5 & 130 & 29 & 3 & 6 & 2 & 0 & 32 & 4 & 30 & 25 & 1 & $<10$ \\
\hline 14 & Mozn & $7-7.6$ & $120-150$ & 18 & 2 & 10 & 5 & 0 & 24 & 2 & 7 & 65 & 1 & $<10$ \\
\hline 15 & Aquafina & 7 & 110 & 16 & 1 & $<5$ & 13 & 0.01 & 27.5 & $<0.1$ & 51 & 1.3 & 1 & - \\
\hline 16 & Farm Superstores & 7.2 & 130 & 29 & 1 & 6 & 2 & - & 32 & 4 & 30 & 25 & 1 & $<10$ \\
\hline 17 & Hada & 7.15 & 109 & 20 & 0.8 & 13 & 4 & 0 & 30 & 5 & 20 & 30 & 0.8 & - \\
\hline 18 & Dala & $7-7.6$ & $120-140$ & 15 & 1.7 & 12 & 3 & 0 & 23 & 0.5 & 14 & 24 & 1 & $<10$ \\
\hline 19 & Hayat & 7.2 & 125 & 20 & 1.3 & 10 & 3 & 0.01 & 25 & 6 & 18 & 37 & 0.85 & $<10$ \\
\hline 20 & Hala & 7.2 & 108 & 17.5 & 1 & 10.6 & 4.1 & 0.01 & 2 & 2 & 16 & 55 & 0.7 & - \\
\hline 21 & Alabeer & 7.4 & 115 & 14 & 0.2 & 19 & 2.4 & 0.01 & 45 & 2 & 15 & 22 & 0.9 & - \\
\hline 22 & Hilwa & 7.4 & 185 & 18.9 & 23.5 & 7.1 & 7.9 & 0.01 & 29 & 0 & 45.5 & 84.5 & 0.8 & 0 \\
\hline 23 & Aqua land & 7.2 & 130 & 29 & 1 & 6 & 2 & - & 32 & 1 & 30 & 25 & 1 & $<10$ \\
\hline 24 & Qarzal & 7.2 & 120 & 12.3 & 1.5 & 14.4 & 3 & 0 & 17.5 & 2 & 28 & 24 & 0.9 & - \\
\hline 25 & Najd & 7 & 120 & 16.79 & 1.5 & 10 & 4.45 & 0 & 17 & 3.8 & 35 & 20.04 & 0.8 & - \\
\hline 26 & Najran & 7.4 & 120 & 18 & 1.5 & 19 & 3.5 & - & 13.5 & 3.2 & 27 & 33.5 & 0.6 & - \\
\hline 27 & Hajar & 7.3 & $110-120$ & 10 & 0.25 & 18 & 2.5 & 0 & 33 & 1 & 9 & 45 & 1 & $<10$ \\
\hline 28 & Rafa & $6.5-7.5$ & 110 & 15 & 1 & 12 & 3 & 0 & 14 & 1.5 & 14 & 40 & 1 & $<10$ \\
\hline 29 & Aloyoun & $6.8-7.2$ & $100-130$ & 20 & 1.5 & 10 & 2 & 0.02 & 40 & 5.72 & 5 & 35 & 0.85 & - \\
\hline 30 & Lora & $7-7.4$ & $110-160$ & 22 & 0.68 & 12 & 3 & 0.02 & 31 & 9 & 32 & 13 & 0.9 & - \\
\hline 31 & Al Tharawat & 7.4 & 150 & 2.3 & 0.3 & 33 & 16 & 0.01 & 7 & 1.5 & 12 & 150 & 0.1 & - \\
\hline 32 & Bambini & $6.5-7.5$ & $<50$ & $<3$ & $<0.4$ & $<3$ & $<7$ & $<0.01$ & $<8$ & $<1$ & $<23$ & $<3$ & $<0.5$ & - \\
\hline
\end{tabular}


Table 4. Quality parameter values of imported bottled drinking water brands.

\begin{tabular}{|c|c|c|c|c|c|c|c|c|c|c|c|c|c|c|}
\hline \multirow{2}{*}{ Brand\# } & \multirow{2}{*}{ Brand Name } & \multirow{2}{*}{$\mathrm{pH}$} & \multirow{2}{*}{$\begin{array}{c}\text { TDS } \\
(\mathrm{mg} / \mathrm{L})\end{array}$} & \multicolumn{5}{|c|}{ Cations Average Composition (ppm) } & \multicolumn{5}{|c|}{ Anions Average Composition (ppm) } & \multirow{2}{*}{$\begin{array}{l}(\mathrm{ppb}) \\
\mathrm{BrO}_{3}\end{array}$} \\
\hline & & & & $\mathrm{Na}$ & $\mathrm{K}$ & $\mathrm{Ca}$ & $\mathrm{Mg}$ & $\mathrm{Fe}$ & $\mathrm{Cl}$ & $\mathrm{NO}_{3}$ & $\mathrm{SO}_{4}$ & $\mathrm{HCO}_{3}$ & $\mathrm{~F}$ & \\
\hline 1 & Evian & 7.2 & 309 & 6.5 & 1 & 80 & 26 & - & 6.8 & 3.7 & 12.6 & 360 & - & - \\
\hline 2 & Elite & 7.4 & 120 & 1.2 & 0.3 & 47.6 & 9.9 & - & 1.7 & - & 2.3 & 189.1 & 0.02 & - \\
\hline 3 & Roseland & 7 & 130 & 12 & 6 & 12 & 8 & - & 15 & 7.3 & 9 & 74 & - & - \\
\hline 4 & Tannourine & 7.8 & 170 & 5.6 & 0.7 & 40.5 & 10.1 & - & 6.1 & $<3.1$ & 5.3 & 150 & - & - \\
\hline 5 & Masafi & 7.5 & 140 & 1 & - & 89 & 5.3 & 0.08 & 2.9 & - & 15 & - & - & - \\
\hline 6 & Volvic & 7.4 & 150 & 2.3 & 0.3 & 33 & 16 & $<0.01$ & 7 & 1.5 & 12 & 150 & 0.1 & - \\
\hline 7 & Highland & 7.9 & 170 & 4 & 1 & 50 & 13 & $<0.5$ & 10 & 0.5 & 4 & 160 & $<0.2$ & - \\
\hline 8 & Rim & 6.93 & - & 3.7 & - & 0.3 & 0.06 & - & 4.18 & 0.48 & - & 24.4 & - & - \\
\hline 9 & Hamidiye & 7.96 & 102 & 2.6 & - & 37.5 & 1.7 & - & 1.07 & 3.22 & 5.37 & 122 & 0.12 & - \\
\hline 10 & Oxygenizer & $6.5-7.2$ & $<8$ & $<1$ & $<1$ & $<5$ & $<0.5$ & - & 1 & 1 & $<2$ & 5 & - & - \\
\hline 11 & Fiji & 7.7 & 220 & 18 & 5 & 18 & 15 & - & 9 & 1 & 1 & 153 & - & - \\
\hline 12 & Goccia blu & 7.39 & 331.8 & 18.4 & 2.2 & 98.2 & 3.8 & - & 20 & 3.7 & - & 309 & - & - \\
\hline
\end{tabular}

10) Study of water parameters only concern the excess amounts with respect to standards and not the shortage.

$$
\begin{gathered}
\text { Total Score }=\text { EPA Pts }+ \text { WHO Pts }+ \text { SASO Pts } \\
\text { Normalized Score }=\frac{\text { Required Brand Pts }}{\text { Highest Brand Pts }}
\end{gathered}
$$

\section{Results and Discussion}

The compliance analysis results of local and imported bottled drinking water brands with local and international standards are shown in Table 5 and Table 6 , respectively. It is found that most of the water brands were in compliance with certain parameters such as $\mathrm{pH}$, TDS, $\mathrm{Na}, \mathrm{K}, \mathrm{Ca}, \mathrm{Mg}, \mathrm{Fe}, \mathrm{Cl}, \mathrm{SO}_{4}$ and $\mathrm{F}$. However, the carcinogenic compound $\mathrm{BrO}_{3}$ showed unpromising compliance results in both brands due to insufficient labels data.

Based on our scoring technique, the most local water brands in the western region of Saudi Arabia that were in compliance with the three standards were determined to be Fayha and Hilwa which were indexed \#3 and \#22, respectively, (Table 5, Figure 1 and Figure 2). On the other hand, Volvic brand which was indexed \#6 (Table 6, Figure 1 and Figure 3) was estimated to be the optimum imported water brand in the western region of Saudi Arabia. A comparison between the total scores is shown in Figure 1. Moreover, Figure 2 and Figure 3 confirmed our results for local and imported brands, respectively, by showing the normalized scores of the different brands in which the best brands reserved the highest value of (1).

The $\mathrm{pH}$ of the local and imported bottled waters were almost in the same range and varied between $6.5-8.0 \mathrm{mg} / \mathrm{L}$. The local brands TDS values varied 
Table 5. Compliance analysis of different local bottled drinking water brands.

\begin{tabular}{|c|c|c|c|c|c|c|c|c|c|c|c|c|c|c|c|c|}
\hline \multirow[b]{2}{*}{ Brand\# } & \multirow{2}{*}{$\begin{array}{l}\text { Brand } \\
\text { Name }\end{array}$} & \multirow{2}{*}{$\begin{array}{c}\mathrm{pH} \\
(\mathrm{Pts})\end{array}$} & \multirow{2}{*}{$\begin{array}{l}\text { TDS } \\
\text { (Pts) }\end{array}$} & \multicolumn{5}{|c|}{ Cations Average Composition (Pts) } & \multicolumn{5}{|c|}{ Anions Average Composition (Pts) } & \multirow{2}{*}{$\begin{array}{l}\text { (Pts) } \\
\mathrm{BrO}_{3}\end{array}$} & \multirow{2}{*}{$\begin{array}{l}\text { Total } \\
\text { Score } \\
\text { (Points } \\
=\text { Pts) }\end{array}$} & \multirow{2}{*}{$\begin{array}{c}\text { Normalized } \\
\text { Score (\#) }\end{array}$} \\
\hline & & & & $\mathrm{Na}$ & $\mathbf{K}$ & $\mathrm{Ca}$ & $\mathrm{Mg}$ & $\mathrm{Fe}$ & $\mathrm{Cl}$ & $\mathrm{NO}_{3}$ & $\mathrm{SO}_{4}$ & $\mathrm{HCO}_{3}$ & F & & & \\
\hline 1 & $\mathrm{Al}$ Ain & $3,3,3$ & $3,3,3$ & $2,2,2$ & $1,1,0$ & $1,1,1$ & $0,1,1$ & $2,2,2$ & $4,4,4$ & - & $4,4,4$ & $0,0,0$ & $2,2,0$ & $3,3,3$ & 74.00 & 0.96 \\
\hline 2 & Nova & $0,3,3$ & $3,3,3$ & $2,2,2$ & $1,1,0$ & $1,1,1$ & $0,1,1$ & - & $4,4,4$ & - & $4,4,4$ & $0,0,0$ & $2,2,0$ & - & 56.00 & 0.73 \\
\hline 3 & Fayha & $3,3,3$ & $3,3,3$ & $2,2,2$ & $1,1,0$ & $1,1,1$ & $0,1,1$ & $2,2,2$ & $4,4,4$ & - & $4,4,4$ & $0,0,0$ & $2,2,0$ & $4,4,4$ & 77.00 & 1.00 \\
\hline 4 & Panda & $3,3,3$ & $3,3,3$ & $2,2,2$ & $1,1,0$ & $1,1,1$ & $0,1,1$ & - & $4,4,4$ & - & $4,4,4$ & $0,0,0$ & $2,2,0$ & $3,3,3$ & 68.00 & 0.88 \\
\hline 5 & Qtaf & $3,3,3$ & $3,3,3$ & $2,2,2$ & $1,1,0$ & $1,1,1$ & $0,1,1$ & $2,2,2$ & $4,4,4$ & - & $4,4,4$ & $0,0,0$ & $2,2,0$ & $3,3,3$ & 74.00 & 0.96 \\
\hline 6 & Alwadi & $0,3,3$ & $3,3,3$ & $2,2,2$ & $1,1,0$ & $1,1,1$ & $0,1,1$ & $2,2,2$ & $4,4,4$ & - & $4,4,4$ & $0,0,0$ & $2,2,2$ & - & 64.00 & 0.83 \\
\hline 7 & Arwa & $0,3,3$ & $3,3,3$ & $2,2,2$ & $1,1,0$ & $1,1,1$ & $0,1,1$ & $2,2,2$ & $4,4,4$ & - & $4,4,4$ & $0,0,0$ & $2,2,2$ & $3,3,3$ & 73.00 & 0.95 \\
\hline 8 & Nestle & $3,3,3$ & $3,3,3$ & $2,2,2$ & $1,1,0$ & $1,1,1$ & $0,1,1$ & $2,2,2$ & $4,4,4$ & - & $4,4,4$ & $0,0,0$ & $2,2,0$ & $3,3,3$ & 74.00 & 0.96 \\
\hline 9 & Safa & $3,3,3$ & $3,3,3$ & $2,2,2$ & $1,1,0$ & $1,1,1$ & $0,1,1$ & $2,2,2$ & $4,4,4$ & - & $4,4,4$ & $0,0,0$ & $2,2,0$ & $3,3,3$ & 74.00 & 0.96 \\
\hline 10 & Berain & $0,3,3$ & $3,3,3$ & $2,2,2$ & $1,1,0$ & $1,1,1$ & $0,1,1$ & $2,2,2$ & $4,4,4$ & - & $4,4,4$ & $0,0,0$ & $2,2,0$ & $4,4,4$ & 74.00 & 0.96 \\
\hline 11 & Al-Qassim & $3,3,3$ & $3,3,3$ & $2,2,2$ & $1,1,0$ & $1,1,1$ & $0,1,1$ & - & $4,4,4$ & - & $4,4,4$ & $0,0,0$ & $2,2,0$ & - & 59.00 & 0.77 \\
\hline 12 & Tania & $3,3,3$ & $3,3,3$ & $2,2,2$ & $1,1,0$ & $1,1,1$ & $0,1,1$ & $2,2,2$ & $4,4,4$ & - & $4,4,4$ & $0,0,0$ & $2,2,0$ & $3,3,3$ & 74.00 & 0.96 \\
\hline 13 & Hana & $0,3,3$ & $3,3,3$ & $2,2,2$ & $1,1,0$ & $1,1,1$ & $0,1,1$ & $2,2,2$ & $4,4,4$ & - & $4,4,4$ & $0,0,0$ & $2,2,0$ & $3,3,3$ & 71.00 & 0.92 \\
\hline 14 & Mozn & $3,3,3$ & $3,3,3$ & $2,2,2$ & $1,1,0$ & $1,1,1$ & $0,1,1$ & $2,2,2$ & $4,4,4$ & - & $4,4,4$ & $0,0,0$ & $2,2,0$ & $3,3,3$ & 74.00 & 0.96 \\
\hline 15 & Aquafina & $3,3,3$ & $3,3,3$ & $2,2,2$ & $1,1,0$ & $1,1,1$ & $0,1,1$ & $2,2,2$ & $4,4,4$ & - & $4,4,4$ & $0,0,0$ & $2,2,0$ & - & 65.00 & 0.84 \\
\hline 16 & $\begin{array}{c}\text { Farm } \\
\text { Superstores }\end{array}$ & $3,3,3$ & $3,3,3$ & $2,2,2$ & $1,1,0$ & $1,1,1$ & $0,1,1$ & - & $4,4,4$ & - & $4,4,4$ & $0,0,0$ & $2,2,0$ & $3,3,3$ & 68.00 & 0.88 \\
\hline 17 & Hada & $3,3,3$ & $3,3,3$ & $2,2,2$ & $1,1,0$ & $1,1,1$ & $0,1,1$ & $2,2,2$ & $4,4,4$ & - & $4,4,4$ & $0,0,0$ & $2,2,2$ & - & 65.00 & 0.84 \\
\hline 18 & Dala & $3,3,3$ & $3,3,3$ & $2,2,2$ & $1,1,0$ & $1,1,1$ & $0,1,1$ & $2,2,2$ & $4,4,4$ & - & $4,4,4$ & $0,0,0$ & $2,2,0$ & $3,3,3$ & 74.00 & 0.96 \\
\hline 19 & Hayat & $3,3,3$ & $3,3,3$ & $2,2,2$ & $1,1,0$ & $1,1,1$ & $0,1,1$ & $2,2,2$ & $4,4,4$ & - & $4,4,4$ & $0,0,0$ & $2,2,0$ & $3,3,3$ & 74.00 & 0.96 \\
\hline 20 & Hala & $3,3,3$ & $3,3,3$ & $2,2,2$ & $1,1,0$ & $1,1,1$ & $0,1,1$ & $2,2,2$ & $4,4,4$ & - & $4,4,4$ & $0,0,0$ & $2,2,2$ & - & 67.00 & 0.87 \\
\hline 21 & Alabeer & $0,3,3$ & $3,3,3$ & $2,2,2$ & $1,1,0$ & $1,1,1$ & $0,1,1$ & $2,2,2$ & $4,4,4$ & - & $4,4,4$ & $0,0,0$ & $2,2,0$ & - & 62.00 & 0.81 \\
\hline 22 & Hilwa & $0,3,3$ & $3,3,3$ & $2,2,2$ & $0,0,0$ & $1,1,1$ & $0,1,1$ & $2,2,2$ & $4,4,4$ & - & $4,4,4$ & $0,0,0$ & $2,2,2$ & $5,5,5$ & 77.00 & 1.00 \\
\hline 23 & Aqua land & $3,3,3$ & $3,3,3$ & $2,2,2$ & $1,1,0$ & $1,1,1$ & $0,1,1$ & - & $4,4,4$ & - & $4,4,4$ & $0,0,0$ & $2,2,0$ & $3,3,3$ & 68.00 & 0.88 \\
\hline 24 & Qarzal & $3,3,3$ & $3,3,3$ & $2,2,2$ & $1,1,0$ & $1,1,1$ & $0,1,1$ & $2,2,2$ & $4,4,4$ & - & $4,4,4$ & $0,0,0$ & $2,2,0$ & - & 65.00 & 0.84 \\
\hline 25 & Najd & $3,3,3$ & $3,3,3$ & $2,2,2$ & $1,1,0$ & $1,1,1$ & $0,1,1$ & $2,2,2$ & $4,4,4$ & - & $4,4,4$ & $0,0,0$ & $2,2,2$ & - & 67.00 & 0.87 \\
\hline 26 & Najran & $0,3,3$ & $3,3,3$ & $2,2,2$ & $1,1,0$ & $1,1,1$ & $0,1,1$ & - & $4,4,4$ & - & $4,4,4$ & $0,0,0$ & $2,2,2$ & - & 58.00 & 0.75 \\
\hline 27 & Hajar & $3,3,3$ & $3,3,3$ & $2,2,2$ & $1,1,0$ & $1,1,1$ & $0,1,1$ & $2,2,2$ & $4,4,4$ & - & $4,4,4$ & $0,0,0$ & $2,2,0$ & $3,3,3$ & 74.00 & 0.96 \\
\hline 28 & Rafa & $3,3,3$ & $3,3,3$ & $2,2,2$ & $1,1,0$ & $1,1,1$ & $0,1,1$ & $2,2,2$ & $4,4,4$ & - & $4,4,4$ & $0,0,0$ & $2,2,0$ & $3,3,3$ & 74.00 & 0.96 \\
\hline 29 & Aloyoun & $3,3,3$ & $3,3,3$ & $2,2,2$ & $1,1,0$ & $1,1,1$ & $0,1,1$ & $2,2,2$ & $4,4,4$ & - & $4,4,4$ & $0,0,0$ & $2,2,0$ & - & 65.00 & 0.84 \\
\hline 30 & Lora & $3,3,3$ & $3,3,3$ & $2,2,2$ & $1,1,0$ & $1,1,1$ & $0,1,1$ & $2,2,2$ & $4,4,4$ & - & $4,4,4$ & $0,0,0$ & $2,2,0$ & - & 65.00 & 0.84 \\
\hline 31 & Al Tharawat & $0,3,3$ & $3,3,3$ & $2,2,2$ & $1,1,0$ & $1,1,1$ & $0,1,1$ & $2,2,2$ & $4,4,4$ & - & $4,4,4$ & $0,0,0$ & $2,2,2$ & - & 64.00 & 0.83 \\
\hline 32 & Bambini & $3,3,3$ & $3,3,3$ & $2,2,2$ & $1,1,0$ & $1,1,1$ & $0,1,1$ & $2,2,2$ & $4,4,4$ & - & $4,4,4$ & $0,0,0$ & $2,2,2$ & - & 67.00 & 0.87 \\
\hline
\end{tabular}

*Scoring points are reported as the following: (EPA, WHO, SASO $)=(\#, \#, \#)$. 
Table 6. Compliance analysis of different imported bottled drinking water brands.

\begin{tabular}{|c|c|c|c|c|c|c|c|c|c|c|c|c|c|c|c|c|}
\hline \multirow[b]{2}{*}{ Brand\# } & \multirow{2}{*}{$\begin{array}{l}\text { Brand } \\
\text { Name }\end{array}$} & \multirow{2}{*}{$\begin{array}{c}\mathrm{pH} \\
(\mathrm{Pts})\end{array}$} & \multirow{2}{*}{$\begin{array}{l}\text { TDS } \\
\text { (Pts) }\end{array}$} & \multicolumn{5}{|c|}{ Cations Average Composition (Pts) } & \multicolumn{5}{|c|}{ Anions Average Composition (Pts) } & (Pts) & \multirow{2}{*}{$\begin{array}{l}\text { Total } \\
\text { Score } \\
\text { (Points } \\
=\text { Pts) }\end{array}$} & \multirow{2}{*}{$\begin{array}{c}\text { Normalized } \\
\text { Score (\#) }\end{array}$} \\
\hline & & & & $\mathrm{Na}$ & $\mathrm{K}$ & $\mathrm{Ca}$ & $\mathrm{Mg}$ & $\mathrm{Fe}$ & $\mathrm{Cl}$ & $\mathrm{NO}_{3}$ & $\mathrm{SO}_{4}$ & $\mathrm{HCO}_{3}$ & F & $\mathrm{BrO}_{3}$ & & \\
\hline 1 & Evian & $3,3,3$ & $3,3,3$ & $2,2,2$ & $1,1,0$ & $1,1,1$ & $0,1,1$ & - & $4,4,4$ & - & $4,4,4$ & $0,0,0$ & - & $0,0,0$ & 55.00 & 0.86 \\
\hline 2 & Elite & $0,3,3$ & $3,3,3$ & $2,2,2$ & $1,1,0$ & $1,1,1$ & $0,1,1$ & - & $4,4,4$ & - & $4,4,4$ & $0,0,0$ & $2,2,2$ & $0,0,0$ & 58.00 & 0.91 \\
\hline 3 & Roseland & $3,3,3$ & $3,3,3$ & $2,2,2$ & $0,1,0$ & $1,1,1$ & $0,1,1$ & - & $4,4,4$ & - & $4,4,4$ & $0,0,0$ & - & $0,0,0$ & 54.00 & 0.84 \\
\hline 4 & Tannourine & $0,3,3$ & $3,3,3$ & $2,2,2$ & $1,1,0$ & $1,1,1$ & $0,1,1$ & - & $4,4,4$ & - & $4,4,4$ & $0,0,0$ & - & $0,0,0$ & 52.00 & 0.81 \\
\hline 5 & Masafi & $0,3,3$ & $3,3,3$ & $2,2,2$ & - & $1,1,1$ & $0,1,1$ & $2,2,2$ & $4,4,4$ & - & $4,4,4$ & $0,0,0$ & - & $0,0,0$ & 56.00 & 0.88 \\
\hline 6 & Volvic & $0,3,3$ & $3,3,3$ & $2,2,2$ & $1,1,0$ & $1,1,1$ & $0,1,1$ & $2,2,2$ & $4,4,4$ & - & $4,4,4$ & $0,0,0$ & $2,2,2$ & $0,0,0$ & 64.00 & 1.00 \\
\hline 7 & Highland & $0,3,3$ & $3,3,3$ & $2,2,2$ & $1,1,0$ & $1,1,1$ & $0,1,1$ & $0,0,0$ & $4,4,4$ & - & $4,4,4$ & $0,0,0$ & $2,2,2$ & $0,0,0$ & 58.00 & 0.91 \\
\hline 8 & Rim & $0,3,3$ & $3,3,3$ & $2,2,2$ & - & $1,1,1$ & $0,1,1$ & - & $4,4,4$ & - & - & $0,0,0$ & - & $0,0,0$ & 50.00 & 0.78 \\
\hline 9 & Hamidiye & $0,3,3$ & $3,3,3$ & $2,2,2$ & - & $1,1,1$ & $0,1,1$ & - & $4,4,4$ & - & $4,4,4$ & $0,0,0$ & $2,2,2$ & $0,0,0$ & 56.00 & 0.88 \\
\hline 10 & Oxygenizer & $3,3,3$ & $3,3,3$ & $2,2,2$ & $1,1,0$ & $1,1,1$ & $0,1,1$ & - & $4,4,4$ & - & $4,4,4$ & $0,0,0$ & - & $0,0,0$ & 55.00 & 0.86 \\
\hline 11 & Fiji & $0,3,3$ & $3,3,3$ & $2,2,2$ & $0,1,0$ & $1,1,1$ & $0,1,1$ & - & $4,4,4$ & - & $4,4,4$ & $0,0,0$ & - & $0,0,0$ & 51.00 & 0.80 \\
\hline 12 & Goccia blu & $0,3,3$ & $3,3,3$ & $2,2,2$ & $1,1,0$ & $1,1,1$ & $0,1,1$ & - & $4,4,4$ & - & - & $0,0,0$ & - & $0,0,0$ & 40.00 & 0.63 \\
\hline
\end{tabular}

*Scoring points are reported as the following: (EPA, WHO, SASO) $=(\#, \#, \#)$.

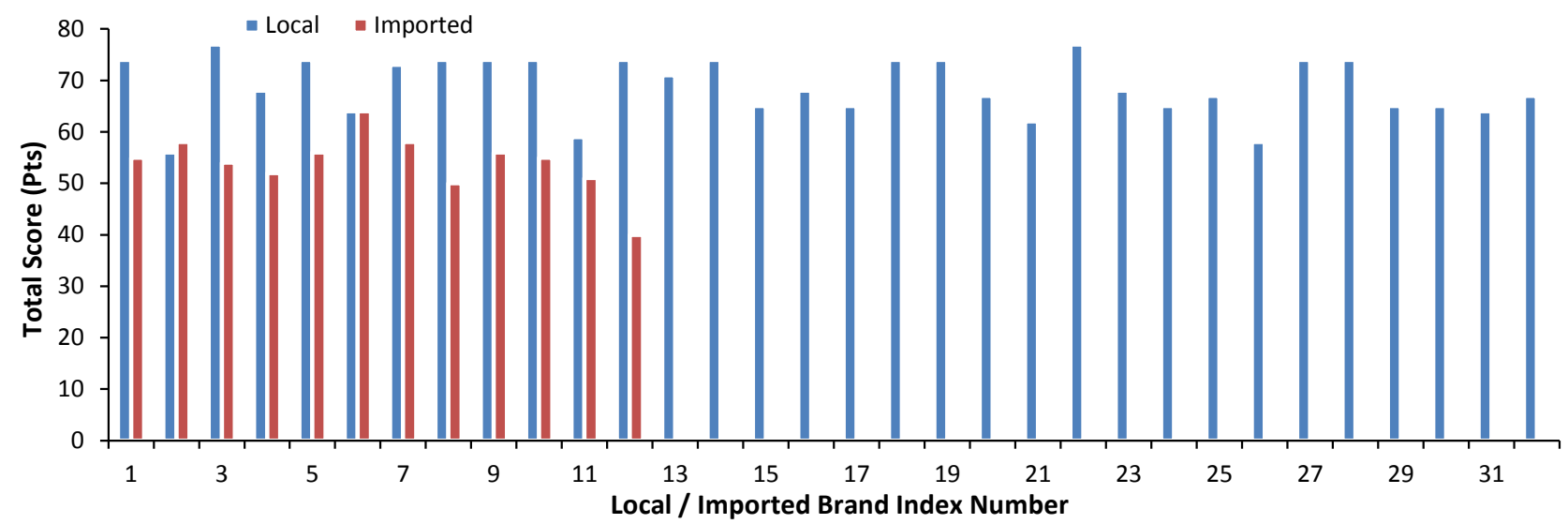

Figure 1. Comparison between total scores of local and imported bottled waters (Brand \# found in Table 5 and Table 6).

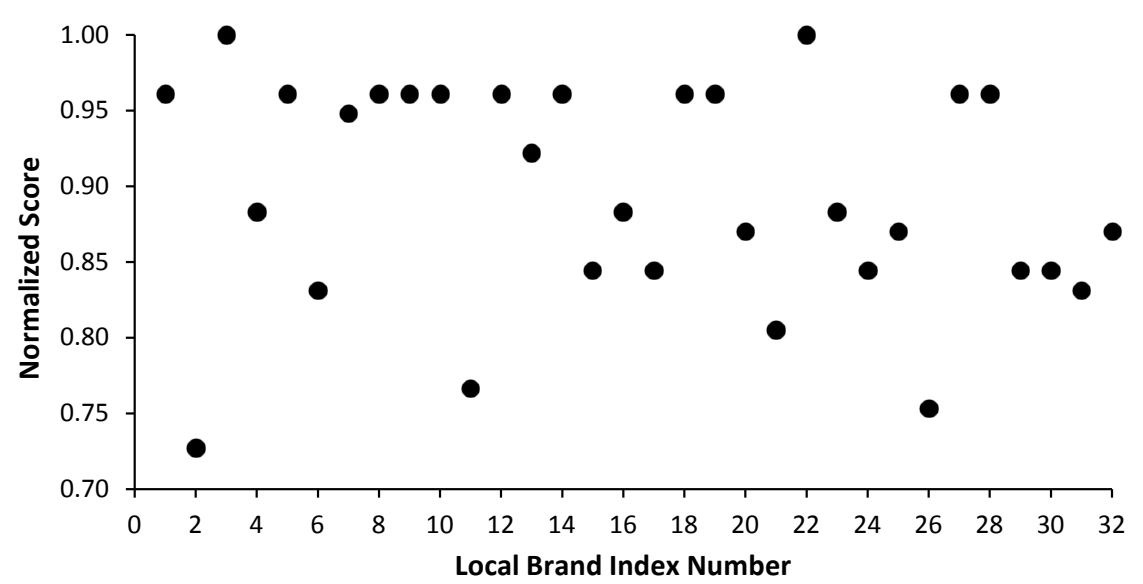

Figure 2. Normalized score results of local bottled waters. 


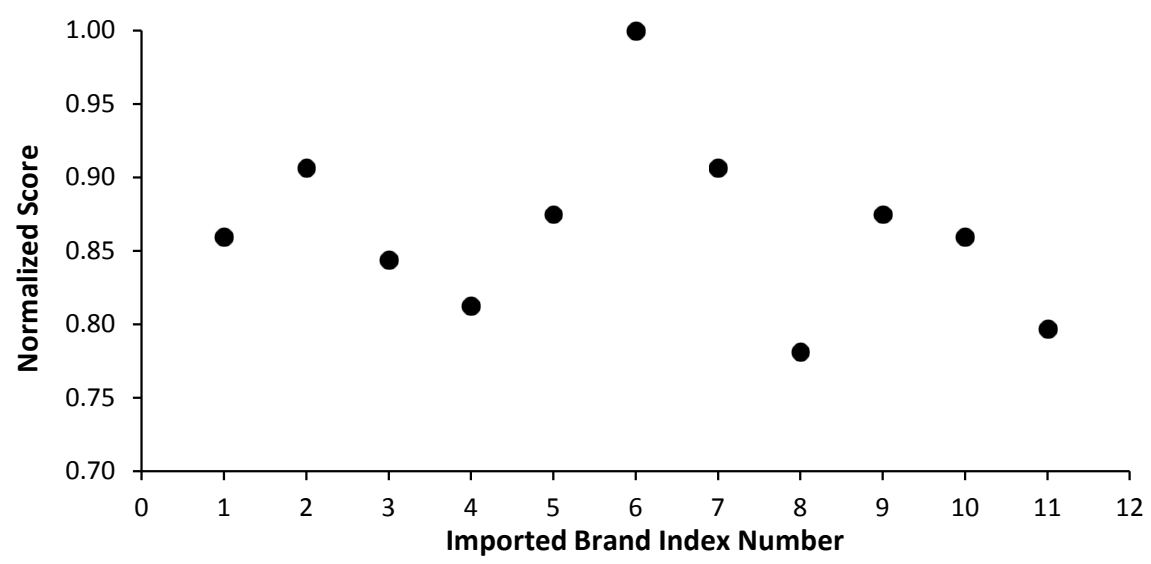

Figure 3. Normalized score results of imported bottled waters.

between $50-185 \mathrm{mg} / \mathrm{L}$ whereas those of the imported ones ranged between 8 $331 \mathrm{mg} / \mathrm{L}$.

Sodium (Na) levels varied between $2.3-29 \mathrm{mg} / \mathrm{L}$ for the local brands and were between 1 - $18.4 \mathrm{mg} / \mathrm{L}$ for the imported brands. As per our studied standards, Sodium should not exceed $95 \mathrm{mg} / \mathrm{L}$ and none of the waters showed a deviation from the suggested value.

Potassium levels in the local brands were between $0.2-23.5 \mathrm{mg} / \mathrm{L}$. Despite that Hilwa water was the only local brand that was not in compliance with our $\mathrm{K}$ standards, it still got the highest score and selected as one of the optimal local brands since it satisfied the three standards of the carcinogenic compound $\mathrm{BrO}_{3}$. However, the maximum $\mathrm{K}$ value registered for the imported brands was 2.2 $\mathrm{mg} / \mathrm{L}$.

The three studied standards recommended a Calcium level in the range of or below $100-200 \mathrm{mg} / \mathrm{L}$. The minimum and maximum Ca concentrations were 1 $36 \mathrm{mg} / \mathrm{L}$ in the local brands and varied between $0.3-98.2 \mathrm{mg} / \mathrm{L}$ in the imported brands. Thus, all the brands satisfied our standards.

The minimum and maximum Magnesium concentrations of the local brands were $0.5 \mathrm{mg} / \mathrm{L}$ and $21.1 \mathrm{mg} / \mathrm{L}$, respectively. SASO (2009) recommended a level of $\mathrm{Mg}$ to be around $150 \mathrm{mg} / \mathrm{L}$ whereas WHO (2013) recommended a value that was approximately $50 \mathrm{mg} / \mathrm{L}$ as shown in Table 2 . The imported $\mathrm{Mg}$ levels ranged between $0.5-26 \mathrm{mg} / \mathrm{L}$.

Iron concentrations in both local and imported (available data) brands were in compliance with our three standards of $0.3 \mathrm{mg} / \mathrm{L}$. Most of the Fe levels in the local brands were between $0.01-0.1 \mathrm{mg} / \mathrm{L}$. However, imported brands data were not sufficient for further comparison.

Chloride levels were between $1-68 \mathrm{mg} / \mathrm{L}$ and $1-20 \mathrm{mg} / \mathrm{L}$ for the local and imported brands, respectively. All the water brands were in compliance and below the recommended standards of $\mathrm{Cl}$ of $150-250 \mathrm{mg} / \mathrm{L}$.

Sulfate in the local brands were between $5-74.5 \mathrm{mg} / \mathrm{L}$ which satisfied our $\mathrm{SO}_{4}$ standards (Table 2). $\mathrm{SO}_{4}$ concentrations in the imported brands were in the range $1-12.6 \mathrm{mg} / \mathrm{L}$. 
Fluoride three standards recommended a range equal to or below $0.8-2 \mathrm{mg} / \mathrm{L}$ (Table 2). The local brands had F levels between $0.1-1.2 \mathrm{mg} / \mathrm{L}$ and imported brands information were not sufficient.

According to WHO (2013) standards, it is compulsory to have a Bromate level less than $10 \mathrm{mg} / \mathrm{L}$. However, EPA (2016) and SASO (2009) even recommend much less concentrations of $\mathrm{BrO}_{3}$ of $0.01 \mathrm{mg} / \mathrm{L}$ and $0.025 \mathrm{mg} / \mathrm{L}$, respectively, since Bromate is strongly related to causing cancer in humans. The local brands had $\mathrm{BrO}_{3}$ levels between $5-10 \mathrm{mg} / \mathrm{L}$ and imported brands information were not sufficient.

Since Fayha and Hilwa brands got the highest score points, they have been selected as the best waters among our studied local and imported waters in the western region of Saudi Arabia; It is worth to check our results by obtaining their scores from the data that is given by Ghrefat in his recent study in 2013 [7]. Table 7 and Table 8 show the comparisons in the total score results between our collected data and Ghrefat data for both brands. It has been proved that all the information are almost identical which lead to the same scores except for Bromate parameter. The difference between both scores is due to the unknown Bromate levels in Ghrefat datasheets. In other words, Fayha and Hilwa brands would not have been selected if Bromate standards were not met.

Generally, the local water brands were more reliable to the studied standards than the imported brands. A lab study was conducted in 2010 to check for the credibility of the reported parameters data in twenty one different local brands that were collected from the supermarkets of Riyadh. The average concentration level of $\mathrm{F}, \mathrm{Ca}$, and $\mathrm{pH}$ were found as $0.86 \mathrm{mg} / \mathrm{L}, 38.47 \mathrm{mg} / \mathrm{L}$, and $7.5 \mathrm{mg} / \mathrm{L}$, respectively, which were higher than the average concentrations reported in the labels. However, the average TDS concentration was found as $118.87 \mathrm{mg} / \mathrm{L}$ which was lower than average concentrations reported on the labels [14]. Various observed levels of $\mathrm{pH}$, TDS and Ca pose no obvious health impacts since they were in or below the recommended range and will have no change to our findings. Yet, Akpata et al. (1997) suggested that having F levels that are higher than $0.8 \mathrm{mg} / \mathrm{L}$, which was observed to be significantly higher than the reported data on the labels in the previous lab study (2010), may develop objectionable fluorosis to children [15]. Authors also claimed that F levels were not in or below the recommended WHO and SASO standard limits [14] [15]. Nonetheless, a single change in parameters values such as $\mathrm{F}$ which got only two score points (Table 2) may not affect our final results of predicting the ideal water brands.

The periodical quality analysis of potable waters is essential to ensure the safety of drinking water to humans. A previous quality assessment study were conducted in Turkey and results showed that a significant number of bottled water brands contained $\mathrm{Na}, \mathrm{Cl}, \mathrm{SO}_{4}, \mathrm{~F}$, polycyclic aromatic hydrocarbons, and several heavy metals were above the maximum allowed levels [16]. However, this study showed that most of the physical and chemical contents of the studied water brands in the western region of Saudi Arabia were found within or below the 
Table 7. Fayha brand scoring comparison.

\begin{tabular}{ccccc}
\hline Parameter & Ghrefat (2013) & This study & Ghrefat score* $^{*}$ & This study score $^{*}$ \\
\hline $\mathrm{pH}$ & 7 & 7.3 & $3,3,3$ & $3,3,3$ \\
$\mathrm{TDS}(\mathrm{mg} / \mathrm{L})$ & 110 & 120 & $3,3,3$ & $3,3,3$ \\
$\mathrm{Ca}(\mathrm{mg} / \mathrm{L})$ & 15 & 15 & $1,1,1$ & $1,1,1$ \\
$\mathrm{Mg}(\mathrm{mg} / \mathrm{L})$ & 4 & 4 & $0,1,1$ & $0,1,1$ \\
$\mathrm{Na}(\mathrm{mg} / \mathrm{L})$ & 13 & 15 & $2,2,2$ & $2,2,2$ \\
$\mathrm{~K}(\mathrm{mg} / \mathrm{L})$ & 0.9 & 0.9 & $1,1,0$ & $1,1,0$ \\
$\mathrm{HCO}(\mathrm{mg} / \mathrm{L})$ & 12 & 15 & $0,0,0$ & $0,0,0$ \\
$\mathrm{SO} \mathrm{S}_{4}(\mathrm{mg} / \mathrm{L})$ & 50 & 20 & $4,4,4$ & $4,4,4$ \\
$\mathrm{Cl}(\mathrm{mg} / \mathrm{L})$ & 14 & 25 & $4,4,4$ & $4,4,4$ \\
$\mathrm{~F}(\mathrm{mg} / \mathrm{L})$ & 0.9 & 0.9 & $2,2,0$ & $2,2,0$ \\
$\mathrm{BrO}_{3}(\mathrm{ppb})$ & - & $<5$ & $0,0,0$ & $4,4,4$ \\
$\mathrm{Fe}(\mathrm{mg} / \mathrm{L})$ & 0.02 & 0.01 & $2,2,2$ & $2,2,2$ \\
$\mathrm{Total} \mathrm{Score} \mathrm{(Pts)}$ & - & - & 65 & 77 \\
\hline
\end{tabular}

*Scoring pts are reported as the following: $(\mathrm{EPA}, \mathrm{WHO}, \mathrm{SASO})=(\#, \#, \#)$.

Table 8. Hilwa brand scoring comparison.

\begin{tabular}{ccccc}
\hline Parameter & Ghrefa $(2013)$ & This study & Ghrefat score* & This study score* $^{*}$ \\
\hline $\mathrm{pH}$ & 7.4 & 7.4 & $0,3,3$ & $0,3,3$ \\
$\mathrm{TDS}(\mathrm{mg} / \mathrm{L})$ & 210 & 185 & $3,3,3$ & $3,3,3$ \\
$\mathrm{Ca}(\mathrm{mg} / \mathrm{L})$ & 28.5 & 7.1 & $1,1,1$ & $1,1,1$ \\
$\mathrm{Mg}(\mathrm{mg} / \mathrm{L})$ & 11.9 & 7.9 & $0,1,1$ & $0,1,1$ \\
$\mathrm{Na}(\mathrm{mg} / \mathrm{L})$ & 23.7 & 18.9 & $2,2,2$ & $2,2,2$ \\
$\mathrm{~K}(\mathrm{mg} / \mathrm{L})$ & 13.4 & 23.5 & $0,0,0$ & $0,0,0$ \\
$\mathrm{HCO}(\mathrm{mg} / \mathrm{L})$ & 120 & 84.5 & $0,0,0$ & $0,0,0$ \\
$\mathrm{SO} \mathrm{H}_{4}(\mathrm{mg} / \mathrm{L})$ & 47.4 & 45.5 & $4,4,4$ & $4,4,4$ \\
$\mathrm{Cl}_{(\mathrm{mg} / \mathrm{L})}$ & 32 & 29 & $4,4,4$ & $4,4,4$ \\
$\mathrm{~F}(\mathrm{mg} / \mathrm{L})$ & 0.8 & 0.8 & $2,2,2$ & $2,2,2$ \\
$\mathrm{BrO}_{3}(\mathrm{ppb})$ & - & 0 & $0,0,0$ & $5,5,5$ \\
$\mathrm{Fe}(\mathrm{mg} / \mathrm{L})$ & 0 & 0.01 & $2,2,2$ & $2,2,2$ \\
$\mathrm{Total} \mathrm{Score}(\mathrm{Pts})^{0}$ & - & - & 62 & 77 \\
\hline
\end{tabular}

*Scoring pts are reported as the following: (EPA, WHO, SASO $)=(\#, \#, \#)$.

acceptable limits for drinking water that were addressed by the three international and local standards EPA (2016), WHO (2013) and SASO (2009).

\section{Conclusions}

In many parts of the world, humans have inadequate access to drinking water 
and use sources contaminated with disease or unacceptable levels of toxins or suspended solids [17]. This study was conducted to verify the compliance of bottled water brands in Saudi Arabia with quality standards. A total of Forty four local and imported bottled water brands were collected from supermarkets in the western region of Saudi Arabia for water quality assessment. A proposed novel scoring system has been introduced in order to evaluate the water quality parameters in both brands. Physical and chemical parameters have been investigated to be in compliance with the international and local standards.

Fayha and Hilwa brands have been selected as the best waters among our studied local brands whereas Volvic brand has been recommended to be the optimum imported water brand in the western region of Saudi Arabia. Generally, the local water brands were more reliable to the studied standards than the imported brands. The present work showed that the quality parameters of bottled water brands in Saudi Arabia were found within or below the standards limits of EPA (2016), WHO (2013) and SASO (2009). In other words, licensed water brands in Saudi Arabia were found to have a good water quality which satisfied the quality requirements of the three studied standards.

\section{Acknowledgements}

The authors would like to acknowledge the Saudi Arabian Cultural Mission (SACM) for their continuous support and encouragement to accomplish this work.

\section{References}

[1] Ogbonmwan, S.E. (2011) Water for Life Ireland Campaign: Supporting the United Nation Water Campaign. The 2 nd United Nation Water day, Expo.

[2] Al-Omran, A.M., El-Maghraby, S.E., Aly, A.A., Al-Wabel, M.I., Al-Asmari, Z.A. and Nadeem, M.E. (2013) Quality Assessment of Various Bottled Waters Marketed in Saudi Arabia. Environmental Monitoring and Assessment, 185, 6397-6406. https://doi.org/10.1007/s10661-012-3032-Z

[3] Saleh, M.A., Abdel-Rahman, F.H., Woodard, B.B., Clark, S., Wallace, C., Aboaba, A. and Nance, J.H. (2008) Chemical, Microbial and Physical Evaluation of Commercial Bottled Waters in Greater Houston Area of Texas. Journal of Environmental Science and Health Part A, 43, 335-347. https://doi.org/10.1080/10934520701795400

[4] Krachler, M. and Shotyk, W. (2009) Trace and Ultratrace Metals in Bottled Waters: Survey of Sources Worldwide and Comparison with Refillable Metal Bottles. Science of the Total Environment, 407, 1089-1096. https://doi.org/10.1016/j.scitotenv.2008.10.014

[5] Alam, I. and Sadiq, M. (1988) An Evaluation of Metal Concentrations in Bottled Waters and Their Health Significance. Environmental Technology, 9, 925-930. https://doi.org/10.1080/09593338809384652

[6] Alabdula'aly, A.I. and Khan, M.A. (1999) Chemical Composition of Bottled Water in Saudi Arabia. Environmental Monitoring and Assessment, 54, 173-189. https://doi.org/10.1023/A:1005810900938

[7] Ghrefat, H.A. (2013) Classification and Evaluation of Commercial Bottled Drinking 
Waters in Saudi Arabia. Research Journal of Environmental and Earth Sciences, 5, 210-218.

[8] United States Environmental Protection Agency (2016) Drinking Water Standards. Web, New York.

[9] World Health Organization (2004) Guidelines for Drinking-Water Quality (Vol. 1). Web, Geneva.

[10] United States Environmental Protection Agency (2009) National Primary Drinking Water Regulations, Disinfection Byproduct Contaminant. EPA 816-F-09-004. New York.

[11] Backer, L.C., Esteban, E., Rubin, C.H., Kieszak, S. and McGeehin, M.A. (2001) Assessing Acute Diarrhea from Sulfate in Drinking Water. American Water Works Association Journal, 93, 76-84. http://www.jstor.org/stable/41298029

[12] World Health Organization (2004) Sulfate in Drinking-Water; Background Document for Development of WHO Guidelines for Drinking-Water Quality. Geneva, Switzerland, 4-5.

[13] Global Healing Center (2008) How Does Chlorine in Water Affect My Health? Chlorine, Cancer, and Heart Disease. GHC Online Publications, 2-3. http://www.bioray.com/content/Chlorine.pdf

[14] Khan, N.B. and Chohan, A.N. (2010) Accuracy of Bottled Drinking Water Label Content. Environmental Monitoring and Assessment, 166, 169-176. https://doi.org/10.1007/s10661-009-0993-7

[15] Akpata, E.S., Fakiha, Z. and Khan, N. (1997) Dental Fluorosis in 12 - 15-Year-Old Rural Children Exposed to Fluorides from Well Drinking Water in the Hail Region of Saudi Arabia. Community Dentistry and Oral Epidemiology, 25, 324-327. https://doi.org/10.1111/j.1600-0528.1997.tb00947.x

[16] Güler, C. (2007) Evaluation of Maximum Contaminant Levels in Turkish Bottled Drinking Waters Utilizing Parameters Reported on Manufacturer's Labeling And Government-Issued Production Licenses. Journal of Food Composition and Analysis, 20, 262-272. https://doi.org/10.1016/j.jfca.2006.10.005

[17] Cosgrove, W.J. and Rijsberman, F.R. (2014) World Water Vision: Making Water Everybody's Business. Routledge, Abingdon, 6. 